\title{
LINKAGE DISTRIBUTIONS AND EPISTACY IN QUANTITATIVE INHERITANCE
}

\author{
R. MORLEY JONES \\ A.R.C. Unit of Biometrical Genetics, Department of Genetics, \\ University of Birmingham, England
}

Received I r.iii.6o

A Gharacter whose inheritance is controlled by a polygenic system gives rise to a distribution of phenotypes which, in non-segregating families and in $\mathrm{F}_{2}$ and the first backcrosses $\mathrm{B}_{1}$ and $\mathrm{B}_{2}$, may be described by its cumulants (Fisher, I925). Other generations such as $\mathrm{F}_{3}$, whose members may be classified into different families according to their parentage, require, for the description of their distributions, the use of compound cumulants (Nelder, I959). These are, in $\mathrm{F}_{3}$ and similar

TABLE I

No. of linked loci which must be considered in evaluating cumulants of any generation

\begin{tabular}{|c|c|c|c|c|}
\hline \multirow{2}{*}{$\begin{array}{c}\text { Order of } \\
\text { cumulant }\end{array}$} & \multicolumn{4}{|c|}{ Type of epistacy } \\
\cline { 2 - 5 } & None & 2-factor & 3-factor & 4-factor \\
\hline I & I & 2 & 3 & 4 \\
2 & 2 & 4 & 6 & 8 \\
3 & 3 & 6 & 9 & I2 \\
4 & 4 & 8 & I2 & I6 \\
\hline
\end{tabular}

generations, the cumulants of the family cumulants. There is only one first order compound cumulant which, being the mean of family means, is simply the over-all generation mean. The second order compound cumulants also are familiar from the work of Mather (1949) and Mather and Vines ( $\left.195^{2}\right)$. In $\mathrm{F}_{3}$, the mean of the family variances, $\kappa_{1 \cdot 2}$, is, apart from sampling variation, the $\mathrm{V}_{2 \mathrm{~F} 3}$ of Mather and Vines, while the variance of family means, $\kappa_{2 \cdot 1}$, is $\mathrm{V}_{\mathbf{1 F 3}}$. These, together with the third order compound cumulants, were first studied by Fisher, Immer and Tedin (1932).

In the presence of linkage, provided there is no epistacy, the generation means are independent of the linkage parameters. In the case of second order cumulants (or compound cumulants) it is only necessary to consider the genes two at a time, so that the recombination 
frequencies between pairs of loci give a sufficient description of the linkage distribution. But to evaluate cumulants of the $r$ th order, it is necessary to consider the genes $r$ at a time, and when $r>3$, the recombination frequencies are no longer sufficient to describe the linkage relations.

When there is two-factor epistacy, the number of loci which have to be considered at a time is doubled; when there is three-factor epistacy it is trebled, and so on (table r). The study of the properties of polygenic systems in which linkage is present has hitherto been confined to those problems which involve not more than two loci at a time; that is to say to generation means with no epistacy, variances and covariances with no epistacy, and generation means with twofactor epistacy. These correspond to the part of table I above the broken line, and will be referred to as problems of class I and 2 .

\section{THE LINKAGE DISTRIBUTION}

In order that the more complex problems of this type may be attacked, it is necessary to have a system of parameters which describe completely the linkage relations among an arbitrary set of loci. Geiringer ( 1944 ) proposed to use for this purpose the relative frequencies of the different types of gamete produced by an individual heterozygous at all the loci. This method has two disadvantages. Firstly, the parameters are not all independent of one another since complementary gametic types have the same frequency and the sum of all the frequencies is unity. The number of independent parameters required for $n$ loci is thus $2^{n-1}-\mathrm{I}$. Secondly, the basic set of parameters appropriate to any given set of $r$ loci does not include the corresponding parameters for a subset of those loci. Both these disadvantages are removed, while the symmetry is retained, in the system which will now be proposed.

Let $p_{a b}$ be the frequency of recombination between loci $a$ and $b$. Then $\mathrm{I}-p_{a b}$ is the frequency with which no recombination occurs between $a$ and $b$. In the same way, let $p_{a b c d}$ be defined so that I $-p_{a b c d}$ is the frequency with which no recombination occurs between any of the loci $a b c$ and $d$, and make similar definitions for any even number of loci. The system of parameters so obtained is progressively enlarged, as the number of loci, $r$, increases, by the introduction of additional parameters none of which is redundant; and the whole system, for any $r$, completely specifies the linkage relations. This is clear from table 2. In fact, in general, the total number of parameters is

$$
\left(\begin{array}{l}
r \\
2
\end{array}\right)+\left(\begin{array}{l}
r \\
4
\end{array}\right)+\left(\begin{array}{l}
r \\
6
\end{array}\right)+\ldots=2^{r-1}-\mathrm{I}
$$

where the terms on the left are the numbers of combinations of $r$ things, $2,4,6, \ldots$, at a time respectively. 
With three loci, no new parameters are needed beyond the recombination frequencies. Geiringer showed that if the two homologous sets of alleles are distinguished by the symbols o and I respectively, the gametic frequencies are

and so on.

$$
\begin{aligned}
& l(\mathrm{o}, \mathrm{o}, \mathrm{o})=l(\mathrm{I}, \mathrm{I}, \mathrm{I})=\frac{1}{4}\left(2-p_{a b}-p_{a c}-p_{b c}\right) \\
& l(\mathrm{o}, \mathrm{o}, \mathrm{I})=l(\mathrm{I}, \mathrm{I}, \mathrm{o})=\frac{1}{4}\left(p_{a c}+p_{b c}-p_{a b}\right)
\end{aligned}
$$

TABLE 2

No. of linkage parameters of different types

\begin{tabular}{|c|c|c|c|c|}
\hline \multirow{3}{*}{$\begin{array}{c}\text { No. of loci } \\
(n)\end{array}$} & \multicolumn{2}{|c|}{ No. of parameters of type : } & \multirow{2}{*}{$\begin{array}{c}\text { Total no. of } \\
\text { parameters }\end{array}$} \\
\cline { 2 - 4 } & $p_{a b}$ & $p_{a b c d}$ & $p_{a b c d e f}$ & \\
\hline & & & & \\
\hline 2 & & $\ldots$ & $\ldots$ & 1 \\
3 & 3 & $\ldots$ & $\ldots$ & 3 \\
4 & 6 & $\mathrm{I}$ & $\ldots$ & 7 \\
5 & $\mathrm{I}$ & 5 & $\mathrm{I}$ & 3 \\
6 & $\mathrm{I}$ & $\mathrm{I}$ & 7 & 63 \\
7 & $2 \mathrm{I}$ & 35 & 7 & \\
\hline
\end{tabular}

For four loci they are easily shown to be

$$
\begin{aligned}
& l(\mathrm{o}, \mathrm{o}, \mathrm{O}, \mathrm{o})=l(\mathrm{I}, \mathrm{I}, \mathrm{I}, \mathrm{I})=\frac{1}{2}\left(\mathrm{I}-p_{a b c d}\right) \\
& l(\mathrm{o}, \mathrm{o}, \mathrm{O}, \mathrm{I})=l(\mathrm{I}, \mathrm{I}, \mathrm{I}, \mathrm{o})=\frac{1}{2} p_{a b c d}-\frac{1}{4}\left(p_{a b}+p_{a c}+p_{b c}\right) \\
& l(\mathrm{o}, \mathrm{o}, \mathrm{I}, \mathrm{I})=l(\mathrm{I}, \mathrm{I}, \mathrm{o}, \mathrm{o})=\frac{1}{4}\left(p_{a c}+p_{a d}+p_{b c}+p_{b d}\right)-\frac{1}{2} p_{a b c d}
\end{aligned}
$$

etc.

It is, of course, possible to reduce the number of linkage parameters if some assumptions are made about the crossing-over process. But these appear generally to lead not to a simplification of the formulæ but rather the reverse. For instance, the $\mathrm{F}_{2}$ variance, in the absence of epistacy and dominance is

$$
\frac{1}{2} \sum_{a} d_{a}^{2}+\sum_{a<b}\left(\mathrm{I}-2 p_{a b}\right) d_{a} d_{b} .
$$

If the assumption of no interference is made, then, for any set of loci $a, b, c, \ldots, e, f$ arranged in the order of their occurrence on the linkage map,

$$
\mathrm{I}-2 p_{a f}=\left(\mathrm{I}-2 p_{a b}\right)\left(\mathrm{I}-2 p_{b c}\right) \ldots\left(\mathrm{I}-2 p_{e f}\right)
$$

but the use of these relations to eliminate some of the parameters merely complicates the expression for the variance.

\section{USE OF HAYMAN'S DEVICE}

In problems of class 2, there is no great difficulty in carrying out the calculations since it is only necessary to consider a typical pair of loci. But with problems of higher class, $n$ say, in which sets of $n$ loci have to be considered, the calculations soon get out of hand unless some special artifice is used. A convenient device for this 
purpose when considering crosses derived from two inbred lines which apart from mutation, cannot show multiple allelism, is that due to Hayman (1954, I955). Kempthorne (I957) states that Hayman's device is sometimes useful when there are two alleles per locus and no epistacy. The first of these conditions is essential but it is by no means necessary that there be no epistacy.

Corresponding to each locus, Hayman introduces a variable $\theta$, which can take three possible values I, o, or - I according as the alleles at the locus are homozygous $A$, heterozygous, or homozygous $a$. ( $A, a$ denote the alleles of greater and lesser effect respectively.) For dealing with problems involving linkage, he also defines for each locus a number, $\phi$, such that $\theta^{2}+\phi^{2}=\mathrm{I}$, so that $\phi$, like $\theta$, can take only the values $I, o$, or $-I$, and the signs of the $\phi$ 's are chosen so that for the double heterozygote, $A a B b, \phi_{a} \phi_{b}$ is $+\mathrm{I}$ or $-\mathrm{I}$ when the genes are in the coupling or repulsion phase, respectively.

When there is no epistacy, the mean phenotype corresponding to any genotype, or genotypic value as it has been termed, may now be written

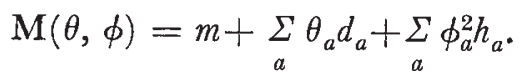

Two-factor epistacy may be allowed for by including terms in $\theta_{a} \theta_{b}$, $\theta_{a} \phi_{b}^{2}, \phi_{a}^{2} \phi_{b}^{2}$ for every pair of loci, the coefficients being the interaction parameters $i_{a b}, j_{a b}, l_{a b}$. Several variants of this notation have been proposed from time to time. These have been discussed by Hayman (I954, I958) and by van der Veen (I959). It is not necessary here to elaborate on this topic, except perhaps to note that if we write

$$
\mathbf{M}(\theta, \phi)=m+\sum_{a} \theta_{a} d_{a}+\sum_{a} \phi_{a}^{2} h_{a}+\sum_{a<b} \theta_{a} \theta_{b} i_{a b}+\sum_{a \neq b} \theta_{a} \phi_{b}^{2} j_{a b}+\sum_{a<b} \phi_{a}^{2} \phi_{b}^{2} l_{a b}
$$

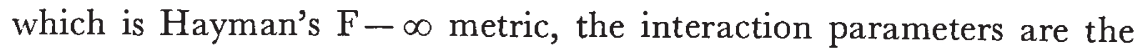
same as those of Hayman and Mather (I955), but the $d_{a}, h_{a}$ correspond in Hayman and Mather's notation to $d_{a}-\frac{1}{2} \sum_{b \neq a} j_{a \mid b}, h_{a}-\frac{1}{2} \sum_{b \neq a} l_{1 a b}$, respectively.

Problems of class $n$ now reduce essentially to the determination of the expected values of products of $\theta$ 's and $\phi$ 's at not more than $n$ loci.

As an example, we evaluate the third cumulant in $\mathrm{F}_{2}$ when there is no epistacy. The moments about the mid-parent are

$$
\begin{aligned}
& \mu_{1}^{\prime}=\sum_{a}\left(h_{a} \mathscr{E} \phi_{a}^{2}\right)
\end{aligned}
$$

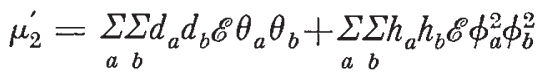

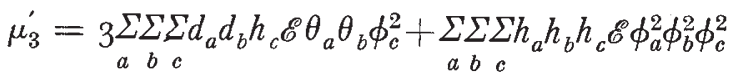

terms of odd degree in $\theta$ and $\phi$ having zero expectations. 
Now it can be shown that

$$
\begin{aligned}
\mathscr{E} \phi_{a}^{2} & =\frac{1}{2} \\
\mathscr{E} \theta_{a} \theta_{b} & =\frac{1}{2}\left(\mathrm{I}-2 p_{a b}\right) \phi_{a} \phi_{b} \\
\mathscr{E} \phi_{a}^{2} \phi_{b}^{2} & =\frac{1}{2}\left(\mathrm{I}-2 p_{a b} q_{a b}\right) \\
\mathscr{E} \theta_{a} \theta_{b} \phi_{c}^{2} & =\frac{1}{2}\left(p_{a c}+p_{b c}-p_{a b}-2 p_{a c} p_{b c}\right) \phi_{a} \phi_{b} \\
\mathscr{E} \phi_{a}^{2} \phi_{b}^{2} \phi_{c}^{2} & =\frac{1}{2}\left(\mathrm{I}-p_{a b} q_{a b}-p_{b c} q_{b c}-p_{a c} q_{a c}\right)
\end{aligned}
$$

where $q=\mathrm{I}-p$, and the $\phi_{a} \phi_{b}$ on the right refers to the linkage phase in $F_{1}$ ( $\pm I$ for coupling and repulsion respectively). Hence after a little reduction,

$$
\begin{aligned}
& \kappa_{1}=\frac{1}{2} \sum_{a} h_{a} \\
& \kappa_{2}=\frac{1}{2} \underset{a b}{\sum \sum} d_{a} d_{b}\left(\mathrm{I}-2 p_{a b}\right) \phi_{a} \phi_{b}+\frac{1}{4} \underset{a b}{\sum \sum} h_{a} h_{b}\left(\mathrm{I}-2 p_{a b}\right)^{2}
\end{aligned}
$$

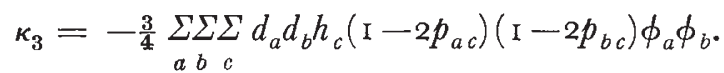

The latter equation obviously reduces to that of Fisher, Immer and Tedin, $\kappa_{3}=-\frac{3}{4} \sum_{a} d_{a}^{2} h_{a}$, when all loci are unlinked. For in that case, all terms are zero, unless $a=b=c$.

\section{SOME GENERAL RESULTS FOR SELFING SYSTEMS}

The general form of the variances in a selfing system was given by Nelder (1952), assuming no epistacy. This is a problem of what we have called the second class. Hayman (1954) also arrived at the solution using the technique outlined above.

When two-factor epistacy is present, the problem becomes one of the fourth class. It is then necessary to evaluate the expectations of products of $\theta$ 's and $\phi$ 's at four loci after $n$ generations of selfing. The generalisation of Nelder's result to this case is a lengthy formula which is probably of limited value. However, the expectations of four-locus products are required for any fourth class problem in a selfing system and are given here for reference. The $\theta$ 's and $\phi$ 's on the l.h.s. of the equations refer to the generation obtained by $n$ successive selfings from that to which r.h.s. refers. No confusion will result from omitting suffices specifying the generation number.

$$
\begin{aligned}
& \mathscr{E}^{n} \phi_{a}^{2} \phi_{b}^{2} \phi_{c}^{2} \phi_{d}^{2}=\alpha^{n} \phi_{a}^{2} \phi_{b}^{2} \phi_{c}^{2} \phi_{d}^{2} \\
& \mathscr{E}^{n} \phi_{a} \phi_{b} \phi_{c} \phi_{d}=\beta^{n} \phi_{a} \phi_{b} \phi_{c} \phi_{d} \\
& \mathscr{E}^{n} \phi_{a} \phi_{b} \phi_{c}^{2} \phi_{d}^{2}=\gamma^{n} \phi_{a} \phi_{b} \phi_{c}^{2} \phi_{d}^{2} \\
& \mathscr{E}^{n} \theta_{a} \theta_{b} \phi_{c}^{2} \phi_{d}^{2}=\left(\frac{1}{2} s_{c d}\right)^{n} \theta_{a} \theta_{b} \phi_{c}^{2} \phi_{d}^{2}-\delta\left\{\frac{1}{2} s_{c d}, \gamma\right\}^{n} \phi_{a} \phi_{b} \phi_{c}^{2} \phi_{d}^{2} \\
& \mathscr{E}^{n} \theta_{a} \theta_{b} \phi_{c} \phi_{d}=\left(\frac{1}{2} r_{c d}\right)^{n} \theta_{a} \theta_{b} \phi_{c} \phi_{d}-\rho_{a b, c d}\left\{\frac{1}{2} r_{c d}, \beta\right\}^{n} \phi_{a} \phi_{b} \phi_{c} \phi_{d} \\
& \mathscr{E}^{n} \theta_{a} \theta_{b} \theta_{c} \theta_{d}=\theta_{a} \theta_{b} \theta_{c} \theta_{d}+\Sigma^{\prime} t_{a b}\left[\mathrm{I}-\left(\frac{1}{2} r_{a b}\right)^{n}\right] \phi_{a} \phi_{b} \theta_{c} \theta_{d} \\
& \quad+\left[\beta\{\mathrm{I}, \beta\}^{n}+\Sigma^{\prime}\left[t_{a b} \rho_{a b, c d}\left(\left\{\beta, \frac{1}{2} r_{a b}\right\}^{n}-\{\mathrm{I}, \beta\}^{n}\right)\right]\right] \phi_{a} \phi_{b} \phi_{c} \phi_{d}
\end{aligned}
$$

where $\Sigma^{\prime}$ denotes summation over the six ways of choosing two out of 
$a, b, c$ and $d$; the notation $\{x, y\}^{n}$ denotes $\left(x^{n}-y^{n}\right) /(x-y)$ (or $n x^{n-1}$ if $x=y$ ); and

$$
\begin{gathered}
\alpha=\frac{1}{2}-p_{a b c d}+\frac{1}{4} \Sigma^{\prime} p_{a b}^{2}+\frac{1}{4}\left(\Sigma^{\prime} p_{a b}-4 p_{a b c d}\right)^{2} \\
\beta=\frac{1}{2}-p_{a b c d}+\frac{1}{2}\left(p_{a b} p_{c d}+p_{a c} p_{b d}+p_{a d} p_{b c}\right) \\
\gamma=\frac{1}{2}-r_{c d} p_{a b c d}+\frac{1}{2}\left(p_{a c} p_{b c}+p_{a d} p_{b d}\right)-\frac{1}{2} p_{c d} \Sigma^{\prime} p_{a b} \\
\delta=r_{c d} p_{a b c d}+\frac{1}{2}\left(p_{a b}+p_{c d}+p_{a c} p_{b c}+p_{a d} p_{b d}\right)-\frac{1}{2} q_{c d} \Sigma^{\prime} p_{a b} \\
\rho_{a b, c d}=p_{a b c d}-\frac{1}{2}\left(p_{a c}+p_{a d}+p_{b c}+p_{b d}-p_{a c} p_{b d}-p_{a d} p_{b c}+p_{a b} p_{c d}\right) \\
q_{a b}=\mathrm{I}-p_{a b}, \quad r_{a b}=\mathrm{I}-2 p_{a b}, \quad s_{a b}=\mathrm{I}-2 p_{a b} q_{a b}, \quad t_{a b}=r_{a b} /\left(\mathrm{I}+2 p_{a b}\right)
\end{gathered}
$$

It is easily shown that $\mathscr{E}^{n} \theta=\theta$ and $\mathscr{E}^{n} \phi=0$ (Hayman, 1955). Other results for less than four loci can be deduced as special cases from equations $(\mathrm{A})$ to $(\mathrm{F})$.

For example, to obtain $\mathscr{E}^{n} \theta_{a} \theta_{b} \phi_{c}^{2}$, let $c$ and $d$ coincide in equation (E). Then if we write $p_{a c}+p_{b c}-2 p_{a c} p_{b c}=p_{a b}$, we find

Hence

$$
\begin{aligned}
& p_{c d}=\mathrm{o}, \quad r_{c d}=\mathrm{I}, \quad p_{a b c d}=\frac{1}{2}\left(p_{a b}+p_{a c}+p_{b c}\right) \\
& \beta=\frac{1}{2}\left(\mathrm{I}-p_{a b}-p_{a b, c}\right), \quad \rho_{a b, c d}=\frac{1}{2}\left(p_{a b}-p_{a b, c}\right) .
\end{aligned}
$$

$$
\mathscr{E}^{n} \theta_{a} \theta_{b} \phi_{c}^{2}=2^{-n} \theta_{a} \theta_{b} \phi_{c}^{2}-2^{-n}\left[\mathrm{I}-\left(\mathrm{I}-p_{a b}-p_{a b, c}\right)^{n}\right] \frac{p_{a b}-p_{a b, c}}{p_{a b}+p_{a b, c}} \phi_{a} \phi_{b} \phi_{c}^{2} .
$$

Putting $n=\mathrm{I}$, and on the r.h.s. all $\theta^{\prime} \mathrm{s}=\mathrm{o}$ and $\phi$ 's $=\mathrm{I}$, we obtain the result for $\mathrm{F}_{2}$ used in the last section.

Again, to obtain $\mathscr{E}^{n} \theta_{b} \phi_{c}^{2}$, let the $a$ locus be unlinked with $b$ and $c$ so that $p_{a b}=p_{a c}=\frac{1}{2}$ and $\theta_{a}, \phi_{a}$ are statistically independent of the other $\theta$ 's and $\phi^{\prime}$ 's. Then $p_{a b, c}=\frac{1}{2}$, and

whence

$$
\mathscr{E}^{n} \theta_{a} \theta_{b} \phi_{c}^{2}=\mathscr{E}^{n} \theta_{a} \cdot \mathscr{E}^{n} \theta_{b} \phi_{c}^{2}=2^{-n} \theta_{a} \theta_{b} \phi_{c}^{2}
$$

$$
\mathscr{E}^{n} \theta_{b} \phi_{c}^{2}=2^{-n} \theta_{b} \phi_{c}^{2}
$$

\section{SUMMARY}

Problems of determining the cumulants of the heritable variation of any generation when linkage and epistacy are both present can be classified according to the number of loci which it is necessary to consider at a time, and among which the linkage relations have to be specified. When this number is greater than three, the recombination frequencies are no longer sufficient to describe the linkage relations, but the system of linkage parameters can be completed by introducing in addition, for every set of an even number of loci, the frequency with which recombination occurs between at least one pair.

The use of Hayman's device in this type of problem is illustrated by obtaining the third cumulant in $\mathrm{F}_{2}$ when there is no epistacy, a problem of class three. Formulæ are given which make possible the 
solution of any problem of class not greater than four, in any generation obtained by repeated selfing after an initial cross between two inbred lines.

\section{REFERENCES}

FISHER, R. A. 1925. Statistical Methods for Research Workers. Oliver and Boyd, Edinburgh. Twelfth edition, 1954 .

FISHER, R. A., IMMER, F. R., AND TEDIN, O. 1932. The genetical interpretation of statistics of the third degree in the study of quantitative inheritance. Genetics, I7, $107-124$.

GEIRINGER, H. 1944. On the probability theory of linkage in Mendelian heredity. Ann. Math. Statist., I5, 25-57.

HAYMAN, B. 1. 1954. A mathematical theory of gene action and interaction. $\mathrm{Ph} . \mathrm{D}$. thesis, Birmingham University Library, Birmingham, England.

HAYMAN, B. I. 1955. The description and analysis of gene action and interaction. Cold Spring Harbor Symp. Quant. Biol., 20, 79-86.

HAYMAN, B. 1. 1958. The separation of epistatic from additive and dominance variation in generation means. Heredity, 12, 371-390.

HAYMAN, B. I., AND MATHER, K. 1955. The description of genic interactions in continuous variation. Biometrics, $I I, 69-82$.

KEMPTHORNe, O. 1957. An Introduction to Genetic Statistics. New York, Wiley, 1957.

MATHER, K. 1949. Biometrical Genetics. London, Methuen.

MATHER, K., AND VINES, A. 1952. The inheritance of height and flowering time in a cross of Nicotiana rustica. In: Quantitative Inheritance, ed. E. C. Reeve and C. H. Waddington, 49-79. H.M.S.O.

NELDER, J. A. 1952. Some genotypic frequencies and variance components occurring in biometrical genetics. Heredity, 6, 387-394.

NELDER, J. A. 1960. The estimation of variance components in certain types of experiment on quantitative genetics. In: Biometrical Genetics, ed. O. Kempthorne, 139-158. Pergamon Press.

VAN DER VEEN, J. H. 1959. Tests of non-allelic interaction and linkage for quantitative characters in generations derived from two diploid pure lines. Genetica, $30,201-232$. 